\title{
Quest for an open MRI scanner
}

\author{
Franco Bertora ${ }^{*}$ Alice Borceto, Andrea Viale and Giulio Sandini \\ Robotics, Brain and Cognitive Sciences Department, Istituto Italiano di Tecnologia, Genova, Italy
}

\begin{abstract}
A study of the motor cortex during the programming, execution and mental representation of voluntary movement is of great relevance; its evaluation in conditions close to reality is necessary, given the close integration of the visuomotor, sensory feedback and proprioceptive systems, as of yet, a functional Magnetic Resonance Imaging (fMRI) scanner allowing a human subject to maintain erect stance, observe the surroundings and conserve limb freedom is still a dream. The need for high field suggests a solenoid magnet geometry that forces an unnatural posture that affects the results, particularly when the motor cortex is investigated. In contrast in a motor functional study, the scanner should allow the subject to sit or stand, with unobstructed sight and unimpeded movement. Two approaches are presented here to solve this problem. In the first approach, an increased field intensity in an open magnet is obtained lining the "back wall" of the cavity with a sheet of current: this boosts the field intensity at the cost of the introduction of a gradient, which has to be canceled by the introduction of an opposite gradient; The second approach is an adaptation of the "double doughnut" architecture, in which the cavity widens at the center to provide additional room for the subject. The detailed design of this kind of structure has proven the feasibility of the solution.
\end{abstract}

Keywords: Functional magnetic resonance, cerebral activation, motor cortex, driving simulator, magnet design, MRI scanner

\section{Introduction}

Over the past two decades, Magnetic Resonance Imaging (MRI) has asserted itself as a powerful diagnostic imaging mean, siding together with Computed Tomography in brain, soft tissues and cartilage imaging.

In recent years, functional Magnetic Resonance Imaging (fMRI) has been an important addition that allows to evidence which regions of the brain are activated corresponding to specific mental or motor activities.

This modality, which allows to "see" the physiological manifestations of thought, has opened new fields for neuroscience research and clinical practice. In this latter case, the ability to evaluate "a priori" the effects of surgical tumor ablation has made possible interventions for the first time, facing the risk of inducing damages worse than those due to the natural growth of the tumoral mass.

In parallel with MRI widespread diffusion, scanner technology has evolved, chiefly in the directions of ease of use, image quality and reduction of imaging time. Many of these progresses are due to the technological evolution of the magnets, which have become more compact and powerful.

What has not changed with time is the shape of the magnet that, almost invariably, takes the shape of a round tube of about $60-70 \mathrm{~cm}$ in diameter where the patient is located.

\footnotetext{
${ }^{*}$ Corresponding author: Franco Bertora, Robotics, Brain and Cognitive Sciences Department, Istituto Italiano di Tecnologia, Genova, Italy. Tel.: +39010 71781451; Fax: +39010 71781208; E-mail: bertora@iit.it.
} 
The claustrophobic sensation causes problems in a non-negligible part of the population, which is enough to stimulate the industries to devise "open" systems in which the patient is located between two plates, rather than inside the tube. As of today, these systems are rather common but they remain inferior to conventional scanners, being limited in field intensity to a maximum of about $1.2 \mathrm{~T}$ and, therefore, not apt to perform functional analyses that require higher fields.

From the basic research point of view, the functional analysis of the brain while executing motor tasks, carries important contributions to the knowledge of the mechanisms at the base of the planning, the coordination, and the handling of muscular activations. A better knowledge of these still not clear processes carries a relevant weight in the advancement of neurosciences, for the design of more performing and "intelligent" robot systems and is of great interest to the automotive and transportation industry [1-9].

In parallel with fMRI, the technique of diffusion imaging allows the tracing of nervous fiber bundles within the brain [10-14] and thus complements the fMRI information. Both techniques contribute to the recent advances in the study of the resting state behavior of the brain (rs-fMRI) [15], further advancing the knowledge of neural processes.

Under these points of view, the traditional structure of an MRI scanner represents a significant obstacle, because of both the limitation in subjects' movement and the unnatural position to which the subject is constrained.

Anyone who is watching a television show while lying in bed could experience a subtle distortion in the way the scene is perceived: an indication that the perception of space has changed. In the same way, it is licit to suppose that the mechanisms of motor activation could be altered by an environment that is unusual.

The above considerations justify the quest for research oriented, fMRI capable, scanners, in which the subject could maintain a natural position (sitting or erect) with the possibility of direct interaction with the ambient, including an open vision and the capability of manipulating objects in front of him.

In the past, even if several attempts have been made in this direction with different purposes, like those devoted to intraoperative applications [16-23], only one of them was aimed at similar goal, yet it excluded the cerebellum and the encephalic spine trunk, that are the seat of significant motor system related activities [24-27].

These requirements directly impact the conventional technology of magnet fabrication and require looking for new configurations of the field generating elements. The resulting scanner, even if designed as a "one-off" basic research device, might also present advantages in routine clinical practice, provided it could be produced at an acceptable cost.

\subsection{State of the art}

Today, brain functional analysis almost invariably involves functional Magnetic Resonance Imaging (fMRI) [28-32]. fMRI is applicable, in principle, to any magnetic resonance scanner but the requirements for image rate, spatial resolution and sensitivity make it practical only in high field (at least $1.5 \mathrm{~T})$ systems $[25,33,34]$.

The programming, execution and mental representation of voluntary movements are a field of great relevance in neurosciences. The visuomotor, sensory feedback and proprioceptive systems are highly integrated and their evaluation in conditions that closely mimic the reality is of great relevance [3540].

As of today, there hasn't been a scanner capable of fMRI acquisitions on a human that allows the subject to keep an erect position, and that, at the same time, allows sufficient room for the execution of 
simple motor tasks $[25,26,34,41]$. Open MRI scanners are limited to field values around 1T, and their shape accommodates patients only in prone/supine position, leaving little room for limb movements [42].

In contrast, the ideal scanner for a motor functional study should allow the subject to sit or stand in a most natural position, with unimpeded limb movements; the field intensity should be high and the stray field minimal. Of course, the inherent limitations of MRI cannot be overcome: in particular the sensitivity to movement has to be taken into account. In fact, immobility is necessary only in the part of the body (here the brain) that is being imaged. Since the receiving coil/coils must anyway closely surround the head, it is rather easy to devise fixation means to keep the head still while all the other parts of the body are left free.

\section{Design methodologies}

The essence of the design of an MRI magnet is a struggle between the contrasting requirements for a substantial homogeneous volume together with a wide magnet opening, to ease patient and operator access.

In addition, a minimum stray field level is desirable in clinical applications it is required.

\subsection{Technical challenges}

The main design challenge is posed by the very nature of the magnetic field that, by virtue of the Maxwell's equations, cannot be uniform in a volume that is not completely enclosed by a continuous electrical current distribution. Two important principles of static magnetic field generation are that the field intensity produced at any given point by a current loop is given by the gradient of the solid angle subtended by the loop when looking from the point and that, in a region free of currents, the magnetic field can be described (exactly like an electrical field) by the gradient of a scalar function, thus making reference to fictitious "magnetic charges" on both sides of the surfaces enclosed by the current loops.

This explains why the most efficient way of generating an almost uniform magnetic field is to employ a long and narrow solenoid. The central loops furnish the more important contribution (the gradient of the solid angle they cover is maximal) but the uniformity of the generated field is poor, since the gradient varies rapidly. The farther and farther ones contribute less to the overall field but increase its homogeneity, since their solid angle gradient will vary less, conducive to a perfectly uniform field if the length were infinite.

Another approach for the generation of uniform field would be to employ a pair of large diameter loops closely spaced apart. The region between the loops would be the seat of a magnetic field that is almost uniform (since the gradient of the solid angle is small) but, by the same token, not too strong. Again, if the diameter of the loops extends to infinity, the field will be uniform (think of the analogy with the electrical field in a plane plate capacitor).

\subsection{Design strategies}

Given that the solution to the generation of uniform field in an open cavity is physically nonexistent, the problem can be re-stated as an inverse problem that has no exact solution and infinite ways to approach a useful compromise between structure openness and extension of uniform region. 
A traditional approach is to simplify the problem by reducing it from three to two dimensions. A two-dimensional configuration that (when extended in the third dimension) generates a uniform field is selected and then truncated to a finite length. Thus, cylindrical magnets can be seen as derivations of the infinite solenoid and " $\mathrm{C}$ " magnets as derivations of the infinite parallel plate capacitor configuration.

An alternate approach starts from a three-dimensional finite configuration that confines the field and contains a closed cavity where the field is uniform. When the cavity is opened on one or more sides, to allow patient access, uniformity distortions are induced, and stray field arises, but the threedimensional initial configuration introduces a new basic solution.

What follows are examples of application of both approaches and the comparison of their solutions.

\section{An optimized 3-D design}

In 3-D design, the starting point is the definition of the shape and size of the cavity and the homogeneous volume. If a Cartesian frame of reference is chosen and the cavity has a prismatic shape, it can be assumed, without loss of generality, that the field is oriented along one of the axes. Being the field static, the problem can be solved in terms of magnetic charges and scalar magnetic potential; and if the structure is composed of flat face polyhedra, the continuity conditions for the normal and tangential components of $\vec{B}$ and $\vec{H}$ at each interface between different materials can be expressed [4345] by simple relations between the current densities (or magnetic charges) and the face normals; this allows finding a solution to the problem of completely enclosing the homogeneous volume (the full magnet cavity in this case) with blocks of uniformly magnetized material or, what is equivalent, with electrical windings. Patient access is obtained by removing one or more blocks among those that do not support a magnetic flux.

The ensuing field distortions will have to be compensated, in a way not too different from what is done in the traditional approach.

Figure 1 shows an example configuration [46]: in Figure 1(a), the cavity is closed, and the field is everywhere homogeneous; in Figure 1(b), three sides have been removed and, although the field leaks out, it remains essentially confined within the structure.

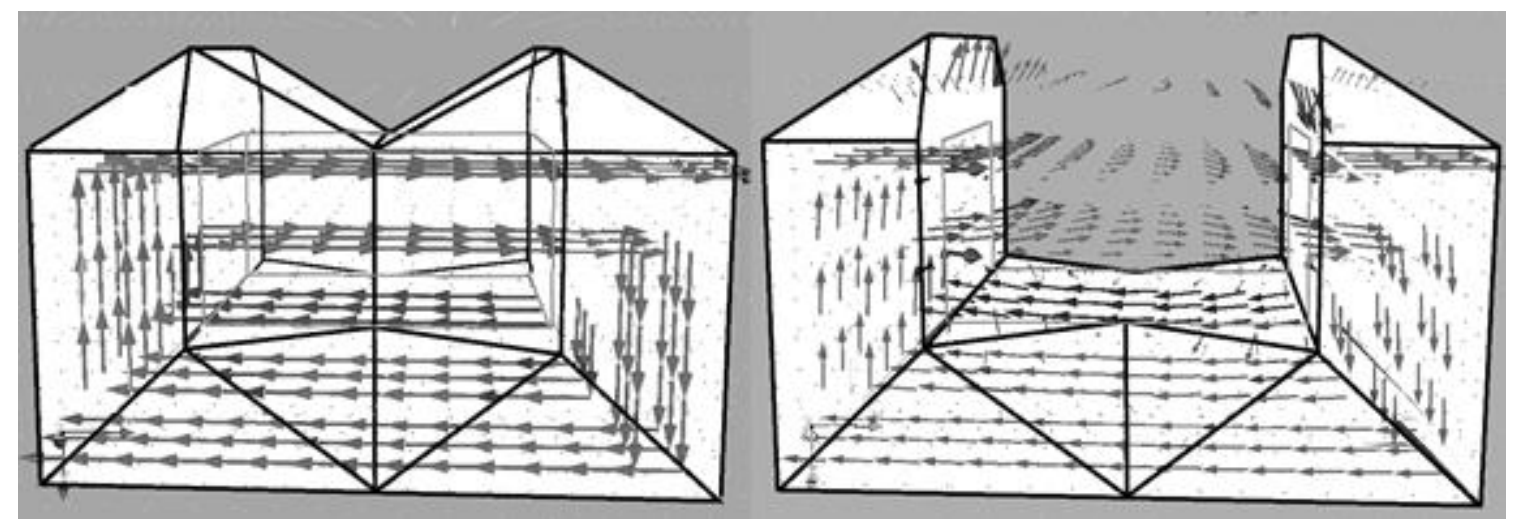

Fig. 1. (a) (left) A closed magnetized structure confining an uniform field within itself and an enclosed cavity (outlined in light color), (b) (right) The same structure in which the cavity has been made accessible by removing portions that do not carry flux. 


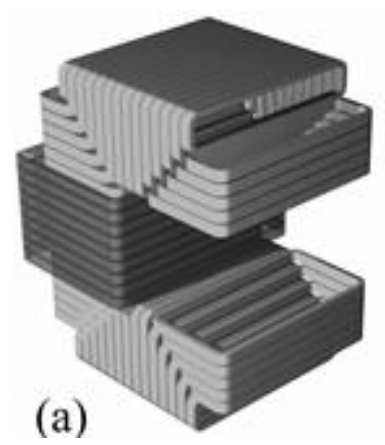

Fig. 2. A three-sided magnet structure (a) and its equivalent in magnetic blocks (b). $\sigma 1$ and $\sigma 2$ represent the magnetic charge densities of the active faces of the dark and light gray blocks.

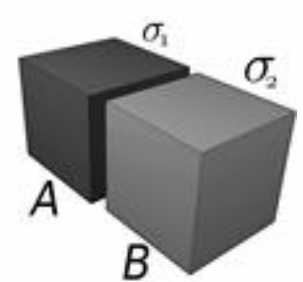

(b)

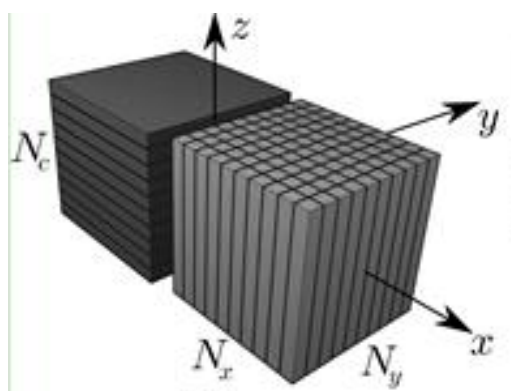

(a)

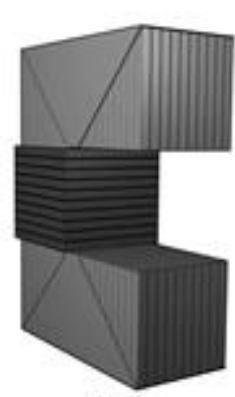

(b)

Fig. 3. The equivalent structure split in elements (a) and a possible real implementation (b).

The effect of the opening introduces a strong distortion of the field, which makes a compensation necessary and shifts the problem to that of generating a complementary field; this can be theoretically effected with elements (coils or permanent magnets) affixed to the faces of the cavity, but it is not practicable with such amount of distortion and a different approach has to be followed.

If the structure in Figure 1 is composed of permanent magnet blocks, even employing modern rare earth materials, it won't be possible to reach a field intensity of at least $1.5 \mathrm{~T}$ : the bare minimum for effective fMRI. It has therefore to be translated to a configuration of electrical currents as, for instance, shown in Figure 2, where the light gray windings take the place of some of the magnetic blocks in Figure 1 [47].

In the structure shown in Figure 2, in Figure 2(a), a section of what is essentially the flux return portion of a "C" magnet is surrounded by a rectangular cross-section coil (dark gray). If the windings are transformed into the equivalent magnetized blocks [45], it can be seen that the resulting magnetic charges assume the configuration in Figure 2(b). In this schematization, one has to remember that the field results from the sum of the $\vec{B}$ field generated by the magnetized block $A$ (the equivalent of the added rectangular cross-section coil) and the $\mu_{0} \vec{H}$ field generated within block $B$ (a fictitious magnetized block occupying the gap of the " $\mathrm{C}$ ").

The homogeneity of the field and the efficiency will depend on a series of parameters as follows.

- The extension of the polar expansion.

- The width of the coil.

- The distance of the polar expansion from the third wall.

- The magnet gap.

- The ratio of average current density in the two main sections of the magnet: the rectangular coil (A), and the "C" (B).

The interaction among these factors can be explored by means of brute force heuristic search [48]. Alternatively, it can be played in a more effective way that exploits the linearity of the problem [49].

The Inverse Boundary Element Method has often been used in the design of gradient or "shim" coils. Given one or more two-dimensional manifolds, over which current paths can be established, and a region of interest (defined by a finite number of points) over which some field properties have to be satisfied, the essence of the method lies in the subdivision of the "active" surfaces in a finite number 
of elements and in the construction of a linear system connecting the contribution of each element to every point defining the region of interest.

In the method as introduced in [50-52], the nodal basis functions are currents flowing in the cluster of elements around the node. Their sum gives rise to a "stream function" whose constant level paths determine the location of the conductors.

In the present case, a similar method can be applied by subdividing the active surfaces of block $B$ in a number of rectangular patches while the winding $A$ is replaced by a stack of rectangular "racetrack" coils, each of which can be reduced to a pair of opposite magnetic charges. The outcome of the process is shown in Figure 3(a) and is equivalent to a number of prismatic permanent magnets. The contribution of the $N_{\mathrm{c}}$ values of the $B_{\mathrm{z}}$ field generated by each of the blocks representing the "racetrack" coils and that of the $N_{\mathrm{ix}} \times N_{\mathrm{y}}$ values of the $\sigma_{0} H_{\mathrm{z}}$ field generated by the elements into which block $B$ has been subdivided, at the $N_{\mathrm{i}}$ points of the region of interest, define a $N_{\mathrm{i}} \times\left(N_{\mathrm{c}}+\left(N_{\mathrm{x}} \times N_{\mathrm{y}}\right)\right)$ matrix. The desired values of the field at the $N_{\mathrm{i}}$ points that define the region of interest make up the right hand side vector of a linear system whose solution yields the magnetic charges necessary to solve the problem.

The conversion from $\left(N_{\mathrm{c}}+\left(N_{\mathrm{x}} \times N_{\mathrm{y}}\right)\right)$ magnetic charges $\sigma_{\mathrm{j}}$ back to currents in the windings is immediate for the $N_{\mathrm{c}}$ "racetrack" coils: the linear current density flowing in each of them is simply given by $\sigma \mu_{0}[\mathrm{~A} / \mathrm{m}]$. In a similar way, the $N_{\mathrm{x}} \times N_{\mathrm{y}}$ charge values on the patches into which top and bottom surfaces of $B$ have been divided can be thought as the ends of elementary coils, adjacent to each other to fill the whole surface. It is easy to see that the currents on adjacent sides of the coils are canceled out, which allows to merge the single coils into larger ones, defined by the constant value paths of the function, in strict analogy to what is done with the stream function.

From the scalar potential point of view, it is seen that the difference between the stream function and the discrete function $\sigma_{\mathrm{j}}(x, y)$ lies in the fact that the stream function describes a surface distribution of current filaments, each equivalent to an $m=\mu_{0} I$ surface magnetic dipole moment distribution, while a pair of opposite surface charge distributions is equivalent to the end surfaces of a coil carrying a surface current of intensity $\sigma / \mu_{0}$.

In practice, these equivalent coils cannot be physically located between the surfaces of $B$, but will have to follow the path of the "C" structure, as shown in Figure 3(b) and in a real implementation will have to be merged into single coils carrying the total flux in the top and bottom horizontal and back vertical tracts of the " $\mathrm{C}$ ". The field distortions thus introduced will have to be evaluated by means of more accurate simulations but, due to their distance from the region of interest and the fact that high spatial frequency field perturbations decay very rapidly with distance, they can be expected to be minor and are here neglected for the sake of simplicity.

\subsection{Results}

What has been described above is applied to a sample structure exhibiting a unit size cubic cavity: a rather extreme example in terms of a gap to other dimensions ratio, which is chosen to stress the capabilities of the method. The objects $A$ and $B$ are therefore unit cubes with centers located at a distance of 0.7 units, leaving a void of size 0.2 units between the two.

If the unit of measure is taken to be $1 \mathrm{~m}$ and the target field intensity is set to $2 \mathrm{~T}$, the resulting structure will fit the reference application of a scanner for functional studies on human subjects in a natural position, with reasonable room for limb movement. In what follows, however, the main accent is on obtaining the desired relative uniformity, rather than on the engineering implications stemming from the necessary current densities. 
The $A$ section is subdivided into 9 equal height "racetrack" coils while section $B$ is subdivided into $7 \times 11$ prismatic sections in the direction of the $x$ and $y$ axes respectively. A cartesian reference system is defined, as indicated in Figure 3(a). The region of interest is an oblate ellipsoid of semi-axes 0.15, $0.15,0.1$ centered around the point $(0.35,0,0)$, uniformly sampled at 16 points on each of 8 parallels located at $\theta=\pi / 16+k \pi / 8$, where $k=0 . .7$ for a total of 128 points

The resulting matrix has size of $128 \times 86$. It is solved recurring to the Moore-Penrose pseudoinverse, regularized by cutting off the less significant singular values of the SVD decomposition [53]. The resulting values of the currents in the coils (for $1 \mathrm{~T}$ field in the region of interest) are shown in Figures 4(a) and 4(b); the corresponding homogeneity over the ellipsoid, as well as the homogeneity over a sphere of diameter 0.2 units, along with the lower terms of a spherical harmonics expansion are found in Table 1.

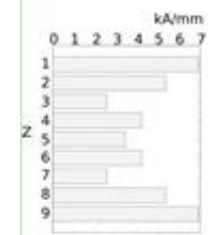

a)

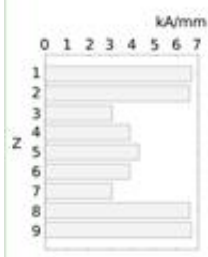

e)
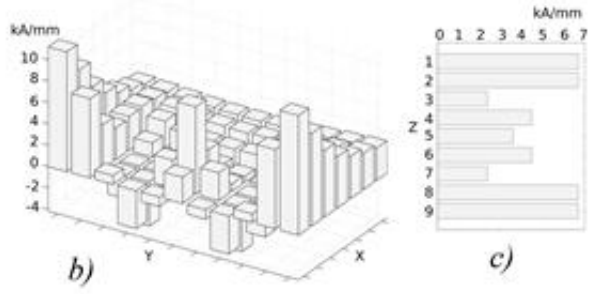

c)

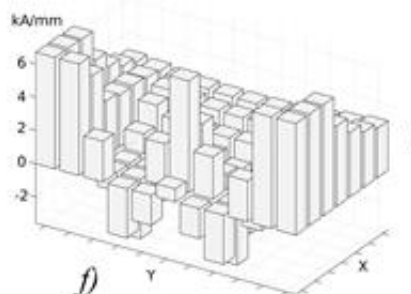

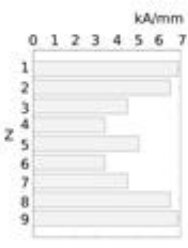

g)
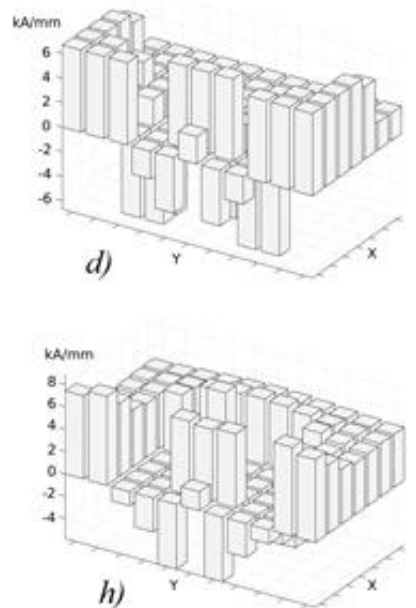

Fig. 4. The equivalent charges on the "racetrack" coils and the prismatic elements, respectively: (a), (b) resulting from a Moore-Penrose pseudo-inverse solution. (c), (d) resulting from a Linear Programming solution. (e), (f) resulting from a single iteration of the "pushing" algorithm applied to the pseudo-inverse solution. (g), (h) resulting from the application of a "null space" correction to the pseudo-inverse solution.

Table 1

Spherical harmonics coeff. (ppm) over a 0.2 diameter sphere

\begin{tabular}{|c|c|c|c|c|c|c|c|c|c|c|c|c|c|}
\hline \multicolumn{8}{|c|}{$\begin{array}{l}\text { PSEUDO-INVERSE SOLUTION } \\
\text { Homog.: } 888.6 \text { (ellipsoid), } 173 \text { ppm (sphere) }\end{array}$} & \multicolumn{6}{|c|}{$\begin{array}{l}\text { LINEAR PROGRAMMING } \\
\text { Homog.: } 678.8 \text { (ellipsoid), } 390 \text { ppm (sphere) }\end{array}$} \\
\hline $1 \backslash \mathrm{m}$ & 0 & 1 & 2 & 3 & 4 & 5 & 6 & $\begin{array}{ll}0 & 1\end{array}$ & 2 & 3 & 45 & & 6 \\
\hline 0 & & 12.6 & 29.8 & -8.7 & 165. & 9.1 & 0.4 & 142. & 221. & -139. & 81.4 & 17.2 & -0.5 \\
\hline 2 & 0 & 0.0 & -28.2 & -40.7 & 83.0 & -59.4 & 12.1 & $\begin{array}{ll}0 & 0.0\end{array}$ & -157 & -177. & 119. & -109. & 6.0 \\
\hline 4 & 0 & 0.0 & 0.0 & 0.0 & 11.4 & -57.6 & 50.8 & $\begin{array}{ll}0 & 0.0\end{array}$ & 0.0 & 0.0 & -95.0 & -122 & 59 \\
\hline
\end{tabular}

\begin{tabular}{|c|c|c|c|c|c|c|c|c|c|c|c|c|c|c|}
\hline \multicolumn{8}{|c|}{$\begin{array}{l}\text { ITERATIVE MODIFICATION } \\
\text { Homog.: } 1697.6 \text { (ellipsoid), } 460 \text { ppm (sphere) }\end{array}$} & \multicolumn{7}{|c|}{$\begin{array}{l}\text { NULL-SPACE ADJUSTED } \\
\text { Homog.: } 1064.8 \text { (ellipsoid), } 452 \text { ppm (sphere) }\end{array}$} \\
\hline $\mathbf{I} \mid \mathrm{m}$ & 0 & 1 & 2 & 3 & 4 & 5 & 6 & 0 & 1 & 2 & 3 & 4 & 5 & 6 \\
\hline $\mathbf{0}$ & & 34.6 & -58.8 & 73.8 & 133. & 13.5 & 0.4 & & 28.2 & 16.9 & -8.2 & 106. & 8.4 & 2.0 \\
\hline 2 & 0 & 0.0 & -112.7 & 94.7 & -61.3 & -87.7 & -2.1 & 0 & 0 & -2.9 & -181. & 122. & -33.5 & -4.8 \\
\hline 4 & 0 & 0.0 & 0.0 & 0.0 & -265 & 8.3 & 75.7 & 0 & 0 & 0.0 & 0.0 & -28.2 & -242 & 96.3 \\
\hline
\end{tabular}


The maximum linear current density in some coils (here $11.2 \times 10^{6} \mathrm{~A} / \mathrm{m}, \sigma=14$ ) might be unfeasible, hence the means is needed to reduce it to more manageable values; this can be achieved by noting that, since the system matrix is not full rank, the solution obtained is only one of the many possible.

The Moore-Penrose pseudo-inverse gives a minimum norm (i.e. minimum total current in the windings) solution, indeed a desirable constraint that could, however, be forfeited in view of other goals, of which physical realizability could be one.

Linear programming has been for many times used in similar contexts [54,55] and is readily applicable to the present case. Choosing to limit the current density to $6.7 \times 10^{6} \mathrm{~A} / \mathrm{m}$ (corresponding to a maximum value $|\sigma|_{\max }=3$ for the charge), one obtains the results shown in Figures 4(c) and 4(d) and the relative values in Table 1.

As evident from Figure 4, the linear programming algorithm has a tendency to force the solution to the prescribed limits as often as possible: this may not be desirable in this case. In fact, abrupt changes in the solution imply, in the case of section $B$, high linear current densities in adjacent coil boundaries must flow in vanishing thickness layers: another obstacle in the way of physical feasibility.

In an attempt to alleviate these drawbacks, two different approaches have been tested, that appear to be of help. The first consists in a gentle "pushing" of the solution towards a more manageable form and is implemented as follows:

1. Start from the pseudo-inverse solution and define the areas where the values exceed a desired value.

2. Saturate the solution to the $\max / \mathrm{min}$ desired value

3. Subtract the field generated by the truncated solution from the target field and save the result as vector $r h s_{1}$.

4. Construct the matrix $\mathbf{M}_{1}$ from the original matrix $\mathbf{M}$ eliminating the columns that correspond to the active elements that have been saturated.

5. Solve for $r h s_{l}$ (again by the pseudo-inverse method), the linear system thus obtained.

6. Combine the new solution with the previous one.

7. Repeat steps from 1 to 6 above until a satisfactory result is obtained. (Beware: no convergence is warranted)

This procedure applied once to the original solution gives the results shown in Figures 4(e) and 4(f) exhibiting a reduction that nearly completely satisfies the requirements (here, as before, set at a maximum linear current density of $6.7 \times 10^{6} \mathrm{~A} / \mathrm{m}$ ) but with significantly less abrupt variations in the solution.

The last approach attempts to shape the result leverages on the fact that the system matrix is not full rank, which implies a non-vanishing extent of the matrix null space, a basis of which is obtained, almost as a by-product, from the singular value decomposition employed in the construction of the pseudo-inverse. By definition, a deficient rank matrix $M$ maps a region of the domain to zero. Any input point lying in this region may be added to any other input point without changing where, in the range, the latter maps. Thus, given any solution, it is possible to add it to any linear combination of null space vectors, without modifying the outcome.

With these premises, the second method goes as follows:

1. Given the SVD decomposition of matrix $\mathbf{M}, \mathbf{M}=\mathbf{U S V}^{\mathrm{T}}$ construct a matrix $\mathbf{O}$ from the columns of $\mathbf{V}$ that correspond to zero (or small) values of diagonal matrix $\mathbf{S}$. Matrix $\mathbf{O}$ is a basis of the null space of $\mathbf{M}$.

2. Define a reasonable shape for the solution and construct a vector $r h s_{0}$ as the difference between the desired solution and the one obtained with any of the previous methods. 
3. Solve the system defined by matrix $\mathbf{O}$ for $r h s_{0}$ and sum the new solution to the old one.

The tricky part in this procedure lies in defining the "reasonable" shape for the solution: if, on one side, it is easy to see what it should be at the points where the original solution exceeds the given limits, there will be no guideline in stating where it should aim elsewhere. The approach that it has been empirically found to work in a number of cases is that of specifying a solution given by limit $_{\text {value }} \times \operatorname{sign}(s)$, where $s$ represents the starting solution. Notice that some experiments are also necessary for fixing limit $_{\text {value, }}$, it has not (as it was in the previous cases) a literal meaning.

Employing the strategy just outlined and fixing limit $_{\text {value }}=2$ (in terms of charges), the results shown in Figures 4 (g) and 4(h) are obtained; they compare favorably with those obtained in other ways.

\section{An optimized 2-D design}

A more traditional 2-D axisymmetrical design can be optimized by means of the proven multi-coil approach, based on the optimization of field homogeneity, flux leakage, maximum current density and conductor length (both of these factors are particularly relevant to superconducting windings), pole diameter and gap size.

Multi-coil design has been often used in the design of conventional magnets [56], and it is easily adapted to the present case. The method consists in solving the inverse problem solution posed by the decomposition of the magnet in a set of coils, and by the search of their optimal dimensions, positions, and currents that produce the desired field.

The magnetic field can be expanded in spherical harmonic functions, both inside and outside spheres enclosing all sources. If the source coils are circular and coaxial, and the sphere is centered on their axis, the field can be expressed in terms of zonal harmonics only [57].

The problem solution amounts to the definition of an allowed volume for the coils, and to its subdivision in a suitable number of candidate solenoids. The harmonic expansion coefficients inside and outside a reference sphere are computed for each and every significant harmonic order and unitary current density in each solenoid. This results in a linear system of equations that, once solved, yields the desired solution.

As in the previous case, the system is under-determined and typically ill-conditioned. A linear programming (LP) solution is preferred here, since it gives an opportunity to inject extra constraints that qualify the solution (i.e., maximal admissible values for the harmonic coefficients, and limit values for coil current density and length).

\subsection{Results}

Setting the field intensity goal to $2 \mathrm{~T}$, and prescribing a spherical uniform region of $25 \mathrm{~cm}$ diameter in a free cavity of $1 \mathrm{~m}$ wide at the position of the subjects' shoulders represents, again, a design compatible with the proposed purpose. In this case, to comply with the hitherto neglected engineering and economic constraints, the allowable coil space was shaped as shown in Figure 5 to obtain an acceptable compromise between cost and feasibility.

The characteristics are shown in detail in Table 2, while Figure 6 shows the extent of the uniform field region and the stray field; the first covers the prescribed $25 \mathrm{~cm}$ diameter sphere with a homogeneity of the same order as that could be obtained by normal manufacturing practices in a room-temperature cavity measuring $80 \mathrm{~cm}$ at its narrowest point, the second is comparable to that of a 
conventional shielded magnet. The details of the magnetic design, covering engineering and constructional details, are presented in [58], and are depicted in Figure 7.

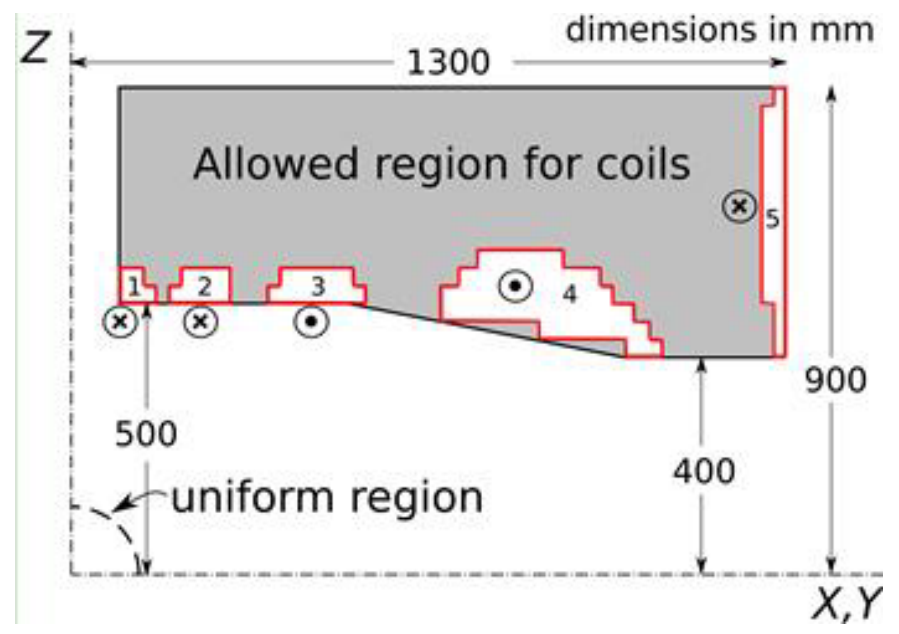

Fig. 5. Allowed regions, resulting coil cross-sections and coils' polarities (only a quarter of the structure is shown).

Table 2

Magnet characteristics

\begin{tabular}{ll}
\hline Room temperature gap & $60-80 \mathrm{~cm}$ \\
Field at center & $2 \mathrm{~T}$ \\
Field uniformity before shimming & $156 \mathrm{ppm}$ over $25 \mathrm{~cm}$ DSV \\
Stray field (5 G line) & $5.3 \times 4.3 \mathrm{~m}$ \\
Stored energy & $31.7 \mathrm{MJ}$ \\
Inductance & $57 \mathrm{H}$ \\
Conductor length & $75.8 \mathrm{~km}$ \\
Current density & $90 \mathrm{~A} / \mathrm{mm}^{2}$ \\
Dimensions & $4 \times 3.8 \times 4.6 \mathrm{~m}(\mathrm{DWH})$ \\
Weight & $60 \mathrm{ton}$ \\
\hline
\end{tabular}
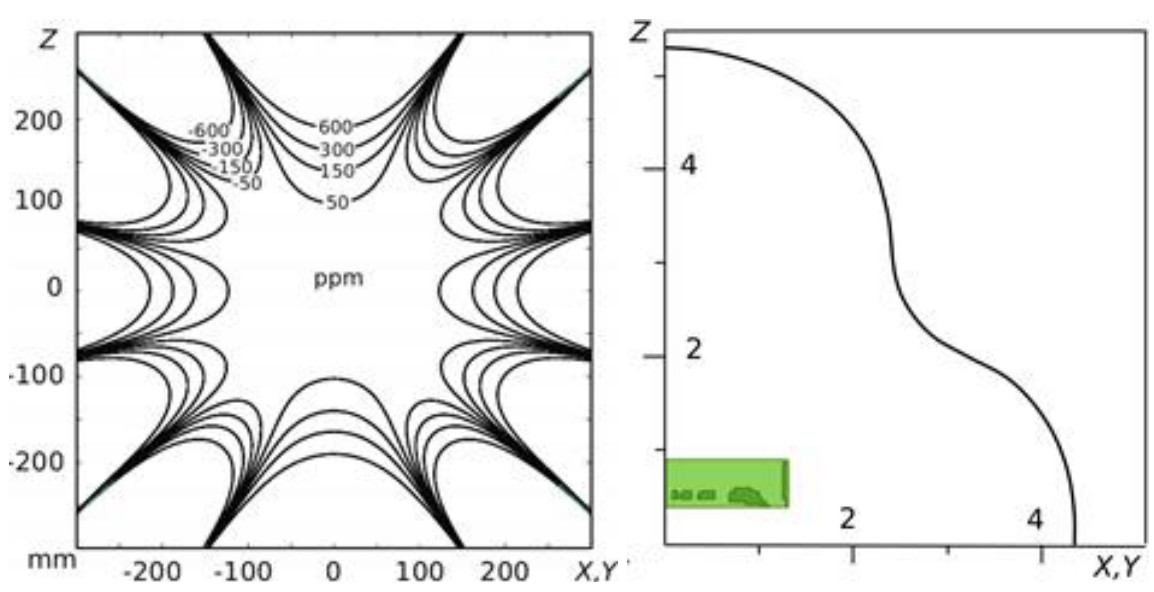

Fig. 6. (a) (left) Field homogeneity in ppm, (b) (right) $5 \mathrm{G}$ stray field line (scale in meters). 


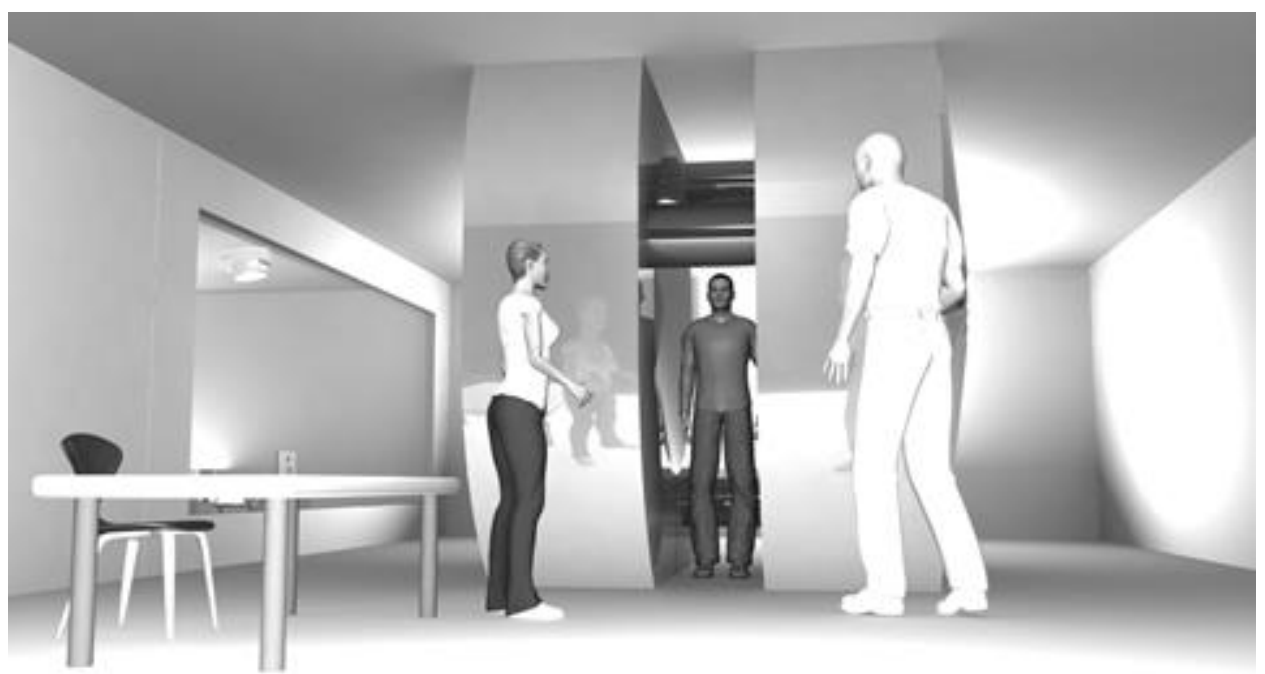

Fig. 7. The magnet easily accommodates a human with ample space for motor activities.

The subject field of view is unrestricted in the vertical direction and is limited to 72 degrees, which decreases to 20 beyond $1 \mathrm{~m}$. In practice, however, the presence of the receiving coil and the necessary fixation means will make it necessary to resort to virtual reality display devices, on the other side, the all-important goal of unimpeded limb movements will be reached.

\section{Conclusion}

The rationale behind a large scale, high field, open MRI scanner aimed at the functional analysis of the human motor cortex has been presented along with two possible realizations.

One of them is based on a novel approach to magnet design that yields the maximum of openness, but is associated with very high costs, deriving from the amount of superconductor employed, and the technological hurdles that mainly stem from the high field intensity on the conductors and the accommodation of the structures needed to sustain them.

The second design is more conventional and has a more restricted cavity, yet it is still satisfactory for the purpose. In addition, it is aligned with the current technology and exhibits a better stray field. The results, obtained in comparing what can be achieved with a diversity of approaches, show the practical feasibility of the magnet design for an open functional MRI scanner.

\section{References}

[1] V.D. Calhoun, J.J. Pekar, T. McGinty, V.B. Adali, T.D. Watson and G.D. Pearlson, Different activation dynamics in multiple neural systems during simulated driving, Human Brain Mapping 16 (2002), 158-167.

[2] F.X. Graydon, R. Young, M.D. Benton, R.J. Genik, S. Posse, L. Hsieh and C. Green, Visual event detection during simulated driving: Identifying the neural correlates with functional neuroimaging, Transportation Research Part F 7 (2004), 271-286.

[3] K. Jahn, A. Deutschländer, T. Stephan, M. Strupp, M. Wiesmann and T. Brandt, Brain activation patterns during imagined stance and locomotion in functional magnetic resonance imaging, NeuroImage 22 (2004), 1722-1731. 
[4] M. Jeong, M. Tashiro, L.N. Singh, M. Yamaguchi, K. Miyake, S. Watanuki, F. Fukuda, T. Takahashi and M. Itoh, Functional neuroimaging of actual car-driving, Cyclotron and Radioisotope Center, Tohoku University Annual Report, $2005,111-114$.

[5] K. Kan, T.A. Schweizer, F. Tam and S.J. Craham, Methodology for functional mri of simulated driving, Medical Physics 40 (2013), 012301-012312.

[6] H.-S. Kim, M.-H. Choi, H.-J. Yoon, H.-J. Kim, U.-H. Jeoung, S.-J. Park, D.-W. Lim, S.-C. Chung and B.-Y. Lee, Cerebral activation and lateralization due to the cognition of a various driving speed difference: An fMRI study, BioMedical Materials and Engineering 24 (2014), 1133-1139.

[7] M. Mader, A. Bresges, R. Topal, A. Busse, M. Forsting and E.R. Gizewski, Simulated car driving in fmri-cerebral activation patterns driving an unfamiliar and a familiar route, Neuroscience Letters 464 (2009), 222-227.

[8] Y. Uchiyama, Drivers' brain activation patterns in car-following situation as determined by functional MRI, Research \& Development Review of Toyota Central R \& D Labs 39 (2004), 47.

[9] Y. Uchiyama, K. Ebe, A. Kozato, T. Okada and N. Sadato, The neural substrates of driving at a safe distance: A functional MRI study, Neuroscience Letters 352 (2003), 199-202.

[10] R. Bammer, Basic principles of diffusion-weighted imaging, Eur. J. Radiol. 45 (2003), 169-184.

[11] D.L. Bihan, J.F. Mangin, C. Poupon, C.A. Clark, N. Pappata, S. Molko and H. Chabriat, Diffusion tensor imaging: Concepts and applications, Journal of Magnetic Resonance Imaging 13 (2001), 534-546.

[12] T.M. Liu, H. Li, K. Wong, A. Tarokh, L. Guo and S.T.C. Wong, Brain tissue segmentation based on DTI data, Neuroimage 38 (2007), 114-123.

[13] Y. Wen and L.H. He, A statistical approach to segmentation of diffusion tensor imaging, Bio-Medical Materials and Engineering 24 (2014), 1253-1259.

[14] S. Westin, C.F. Peled, H. Gudbjartsson, R. Kikinis and F.A. Jolesz, Geometrical diffusion measures for MRI from tensor basis analysis, Proc. Intl. Soc. Mag. Reson. Med. 5 (1997), 1742-1742.

[15] L.H. He, D. Hu, M. Wan and Y. Wen, Measuring temporal dynamics of resting-state fmri data, Bio-Medical Materials and Engineering 24 (2014), 939-945.

[16] B.D. Bai, Y.L. Zhang, D.X. Xie and P.C. Xia, Study on a fully open magnetic resonance imaging device with a slice imaging region, Proceedings of the 2005 IEEE Engineering in Medicine and Biology 27th Annual Conference Shanghai, China 109 (2005), 7008-7011.

[17] F.A. Jolesz, Future perspectives for intraoperative MRI, Neurosurgery Clinics of North America 16 (2005), $201-213$.

[18] Y. Lvovsky and P. Jarvis, Superconducting systems for mri, present solutions and new trends, IEEE Trans. on Appl. Supercond. 15 (2005), 1317-1325.

[19] C. Nimsky, R. Fahlbusch, O. Ganslandt and B. Keller, Intraoperative bildgebung mit 1, 5-Tesla-hochfeldmagnetresonanztomographie, Journal of Neurology, Neurosurgey and Psychiatry 6 (2005), 34-43.

[20] C. Nimsky, A. Fujita, O. Ganslandt, B. von Keller, E. Kohmura and R. Fahlbusch, Frameless stereotactic surgery using intraoperative high-field magnetic resonance imaging, Neurol. Med. Chir. 44 (2004), 522-534.

[21] G.R. Sutherland and D.F. Louw, Intraoperative MRI using a mobile 1.5 T magnet, Techniques in Neurosurgery 7 (2002), 299-307.

[22] Y.Y. Yao, Y.T. Fang, C.S. Koh and G.Z. Ni, A new design method for completely open architecture permanent magnet for MRI, IEEE Transactions on Magnetics 41 (2005), 1504-1507.

[23] Y.Y. Yao, Y.T. Fang, G.Z. Ni and C.S. Koh, The optimal design method of completely open architecture permanent magnet for magnetic resonance imaging, Progress in Electromagnetics Research Symposium, 2005, 23-26.

[24] H. Kitaguchi, O. Ozaki, T. Miyazaki, N. Ayai, K. Sato, S. Urayama and H. Fukuyama. Development of a Bi-2223 hts magnet for 3 T MRI system for human brains, IEEE Trans. Appl. Supercond. 20 (2010), 710-713.

[25] T. Nakada, Clinical experience on 3.0 T systems in niigata, 1996 to 2002, Invest. Radiol. 38 (2003), 377-384.

[26] T. Nakada and N. Tasaka, Human brain imaging in the upright position, Neurology 57 (2001), 1720-1722.

[27] S. Urayama, O. Ozaki, H. Kitaguchi, I. Nakajima, N. Ohnishi, M. Poole, K. Sato and H. Fukuyama, Cryogen-free 3 TMRI system for human brain research using Bi-2223 high-temperature superconducting tapes, Proc. Intl. Soc. Mag. Reson. Med. 20 (2012), 2584-2584.

[28] J. Fridriksson, J. Moss, B. Davis, G.C. Baylis, L. Bonilha and Chris Rordena, Motor speech perception modulates the cortical language areas, NeuroImage 41 (2008), 605-613.

[29] T. Hanakawa, M.A. Dimyan and M. Hallett, Motor planning, imagery, and execution in the distributed motor network: A time-course study with functional MRI, Cereb. Cortex. 18 (2008), 2775-2788.

[30] Y.H. Liu, T.H. Yang, X.H. Yang, I. Liu, W. Liao, S. Lui, X.Q. Huang, H.F. Chen, Q.Y. Gong and D. Zhou, Eeg-fmri study of the interictal epileptic activity in patients with partial epilepsy, Journal of the Neurological Sciences 268 (2001), 117-123. 
[31] A.M. Owen and M.R. Coleman, Functional neuroimaging of the vegetative state, Nat. Rev. Neurosci. 9 (2008), 235243.

[32] M.N. Rajah and A.R. McIntosh, Age-related differences in brain activity during verbal recency memory, Brain Research 1199 (2008), 111-125.

[33] H. Azmi, B. Biswal, S. Salas and M. Schulder, Functional imaging in a low-field, mobile intraoperative magnetic resonance scanner: Expanded paradigms, Neurosurgery 60 (2007), 143-149.

[34] T. Nakada, H. Matsuzawa and I.L. Kwee, High resolution imaging with high and ultra-high-field MRI systems, NeuroReport 19 (2008), 7-13.

[35] Y. Ouchi, H. Okada, E. Yoshikawa, M. Futatsubashi and Shuji Nobezawa, Absolute changes in regional cerebral blood flow in association with upright posture in humans: An orthostatic pet study, The Journal of Nuclear Medicine 42 (2001), 707-712.

[36] Y. Ouchi, H. Okada, E. Yoshikawa, S. Nobezawa and M. Futatsubashi, Brain activation during maintenance of standing postures in humans, Brain 122 (1999), 329-338.

[37] A. Peru and J.S. Morgant, When supine is better than upright: Evidence from postural effects in extinction patients, Brain Research 1110 (2006), 175-181.

[38] A. Raz, B. Lieber, F. Soliman, J. Buhle, J. Posner, B.S. Peterson and M.I. Posner, Ecological nuances in functional magnetic resonance imaging (fMRI): Psychological stressors, posture, and hydrostatics, NeuroImage 25 (2005), 1-7.

[39] M. Stone, G. Stock, K. Bunin, K. Kumar, M. Epstein, C. Kambhamettu, M. Li, V. Parthasarathy and J. Prince, Comparison of speech production in upright and supine position, J. Acoust. Soc. Am. 122 (2007), 532-541.

[40] E.V. Sullivan, J.R. and A. Pfefferbaum, Effect of vision, touch and stance on cerebellar vermian-related sway and tremor: A quantitative physiological and MRI study, Cerebral Cortex 16 (2006), 1077-1086.

[41] T. Nakayama, Y. Fujii, K. Suzuki, I. Kanazawa and T. Nakada, The primary motor area for voluntary diaphragmatic motion identified by high field Fmri, J. Neurol. 251 (2004), 730-735.

[42] D. Hailey, Open magnetic resonance imaging (MRI) scanners, Issues Emerg. Health Technol. 92 (2006), 1-4.

[43] M.G. Abele, Equivalent structures of permanent magnets and electric currents designed to generate uniform fields, J. Appl. Phys. 75 (1994), 6993-6995.

[44] M.G. Abele, Structures of Permanent Magnets: Generation of Uniform Fields, John Wiley \& Sons, New York, USA, 1993.

[45] M.G. Abele, Generation and confinement of uniform magnetic fields with surface currents, IEEE Transactions on Magnetics 41 (2005), 4179-4181.

[46] F. Bertora, E. Molinari and A. Viale, Design strategy for shielded open mri magnets, Proc. Intl. Soc. Mag. Reson. Med. 17 (2009), 3056.

[47] F. Bertora, A. Viale, E. Molinari and P. Fabbricatore, Opening compensation in a $1.5 \mathrm{t}$ open mri magnet for the functional study of the human motor cortex, IEEE Trans. on Appl. Supercond. 20 (2010), 1831-1834.

[48] F. Bertora, P. Fabbricatore, A. Viale and A. Borceto, Three-sided magnets for magnetic resonance imaging, Journal of Applied Physics 109 (2011). doi: 10.1063/1.3537950

[49] F. Bertora, A. Borceto, A. Viale and P. Fabbricatore, A target field approach to open MRI magnet design, IEEE Trans. on Applied Superconductivity 22 (2012). doi: 10.1109/TASC.2011.2178383

[50] R.A. Lemdiasov and R. Ludwig, A stream function method for gradient coil design, Concepts in Magnetic Resonance Part B (Magnetic Resonance Engineering) 26B (2005), 67-80.

[51] G.N. Peeren, Stream function approach for determining optimal surface currents, Journal of Computational Physics 191 (2003), 305-321.

[52] M. Poole and R. Bowtell, Novel gradient coils designed using a boundary element method, Concepts in Magnetic Resonance (Part B), 31B (2007), 162-175.

[53] W.H. Press, B.P. Flannery, S.A. Teukolsky and W.T. Vetterling, Numerical Recipes, The Art of Scientific Computing, Cambridge University Press, New York, USA, 1988.

[54] S. Pittaluga, S. Besio, V. Punzo and A. Trequattrini, Racetrack coils for dedicated MRI magnets, IEEE Trans on Appl. Supercond. 20 (2010), 786-789.

[55] H. Xu, S. Conolly, G.C. Scott and A. Macovski, Homogeneous magnet design using linear programming, IEEE Trans. on Magnetics 36 (2000), 476-483.

[56] M. Kitamura, S. Kakugawa and K. Makiv, An optimal design of coaxial coils with constraints on inner and outer multipole magnetic fields, IEEE Trans. Appl. Supercond. 14 (2004), 1862-1866.

[57] F. Romeo and D.I. Hoult, Magnet field profiling: Analysis and correcting coil design, Magnetic Resonance in Medicine 1 (1984), 44-65.

[58] A. Borceto, D. Damiani, A. Viale, F. Bertora and R. Marabotto, Engineering design of a special purpose functional magnetic resonance scanner magnet, IEEE Trans. on Appl. Supercond. 23 (2013). doi: 10.1109/TASC.2012.2234811 